\title{
Scale effects for strength, ductility, and toughness in "brittle" materials
}

\author{
W.W. Gerberich ${ }^{\text {a) }}$ \\ Department of Chemical Engineering and Materials Science, University of Minnesota, \\ Minneapolis, Minnesota 55455 \\ J. Michler \\ Laboratory for Mechanics of Materials and Nanostructures, EMPA-Swiss Federal Laboratories \\ for Materials Testing and Research, 3602 Thun, Switzerland \\ W.M. Mook \\ Department of Chemical Engineering and Materials Science, University of Minnesota, Minneapolis, \\ Minnesota 55455; and Laboratory for Mechanics of Materials and Nanostructures, EMPA-Swiss \\ Federal Laboratories for Materials Testing and Research, 3602 Thun, Switzerland \\ R. Ghisleni and F. Östlund \\ Laboratory for Mechanics of Materials and Nanostructures, EMPA-Swiss Federal Laboratories \\ for Materials Testing and Research, 3602 Thun, Switzerland \\ D.D. Stauffer \\ Department of Chemical Engineering and Materials Science, University of Minnesota, \\ Minneapolis, Minnesota 55455 \\ R. Ballarini \\ Department of Civil Engineering, University of Minnesota, Minneapolis, Minnesota 55455
}

(Received 11 August 2008; accepted 20 October 2008)

\begin{abstract}
Decreasing scales effectively increase nearly all important mechanical properties of at least some "brittle" materials below $100 \mathrm{~nm}$. With an emphasis on silicon nanopillars, nanowires, and nanospheres, it is shown that strength, ductility, and toughness all increase roughly with the inverse radius of the appropriate dimension. This is shown experimentally as well as on a mechanistic basis using a proposed dislocation shielding model. Theoretically, this collects a reasonable array of semiconductors and ceramics onto the same field using fundamental physical parameters. This gives proportionality between fracture toughness and the other mechanical properties. Additionally, this leads to a fundamental concept of work per unit fracture area, which predicts the critical event for brittle fracture. In semibrittle materials such as silicon, this can occur at room temperature when the scale is sufficiently small. When the local stress associated with dislocation nucleation increases to that sufficient to break bonds, an instability occurs resulting in fracture.
\end{abstract}

\section{INTRODUCTION}

What have become known as the two major-size scale effects in mechanical behavior, the indentation size and grain size effects, are of interest here. However, these are only two of an array of yet-to-be explored phenomena. It is now well understood that decreasing an indentation contact or a nanocrystalline grain size increases measured hardness or strength. ${ }^{1-10}$ There are still questions about the relative importance of gradient plasticity and types of dislocation slip that might be responsible. ${ }^{10,11}$ This has partly been responsible for others showing that nanopillars, ${ }^{12,13}$ columns, ${ }^{14,15}$ or posts $^{16}$ also represent size-scale effects in strength with

\footnotetext{
a) Address all correspondence to this author.

e-mail: wgerb@umn.edu
}

DOI: $10.1557 /$ JMR.2009.0143 smaller volumes representing higher strengths independent of strain gradients. The vast majority of effects have dealt with metals such as $\mathrm{Au},{ }^{1,3} \mathrm{Ni}^{4,9} \mathrm{Cu},{ }^{2,9} \mathrm{Ni}-\mathrm{Fe},{ }^{16}$ and $\mathrm{Al},{ }^{16}$ to cite a few. Besides efforts in the materials science and mechanics communities, there has been increasing interest in the physics, electrical engineering, and mechanical engineering communities because of potential uses of nanodots and nanowires in optoelectronic, interconnect, or MEMS devices. As a result, there has been a flourish of activity to measure length scale effects in the strength, ductility, toughness, and even fatigue of semiconductor and ceramic materials. ${ }^{14-25}$ The intent of this work is to show that there are sizescale effects of strength, ductility, and fatigue measured by others on what have been traditionally considered as truly brittle materials at room temperature. The "brittle" identification in the title is to alert the reader that such 
materials as semiconductors and ceramics may not be truly brittle at all length scales depending on the load case. This is proposed to be due to the intervening appearance of dislocation plasticity prior to fracture during indentation, tension, and compression testing in at least most crystals at small enough size scale. ${ }^{14,15}$ In the following, scale effects for "brittle" materials, the applicability of dislocation shielding, experimental details in silicon, and a theoretically consistent model will be demonstrated.

\section{SCALE EFFECTS FOR MECHANICAL BEHAVIOR OF "BRITTLE" MATERIALS}

Strength, ductility, and fatigue resistance are now found to have scale effects in single crystal nanospheres, micropillars, and nanowires. Several of these are illustrated in Fig. 1. This improvement in properties with decreasing size scale are shown for silicon atomic force microscopy (AFM) tips ${ }^{14}$ having a needle-like shape, uniform silicon nanowires in tension, ${ }^{17}$ and silicon nanospheres in compression. ${ }^{27,28}$ Contact stresses in the $40 \mathrm{GPa}$ ranges accompanied by true fracture strains greater than $100 \%$ are truly remarkable. Also notable is that the AFM tips are the least restrictive in terms of plasticity associated with dislocations as these are small in dimension at the tip but larger in radius away from that tip. The length-scale effect appears to move to larger dimensions for the uniform radius silicon nanowires in tension. ${ }^{17}$ These latter observations, done by direct imaging in a transmission electron microscope, were repeated on $\beta$-SiC with true strains of 0.5 being measured on $86 \mathrm{~nm}$ diameter nanowires. ${ }^{20,23-25}$ The nanosphere observations using both AFM and TEM imaging move the

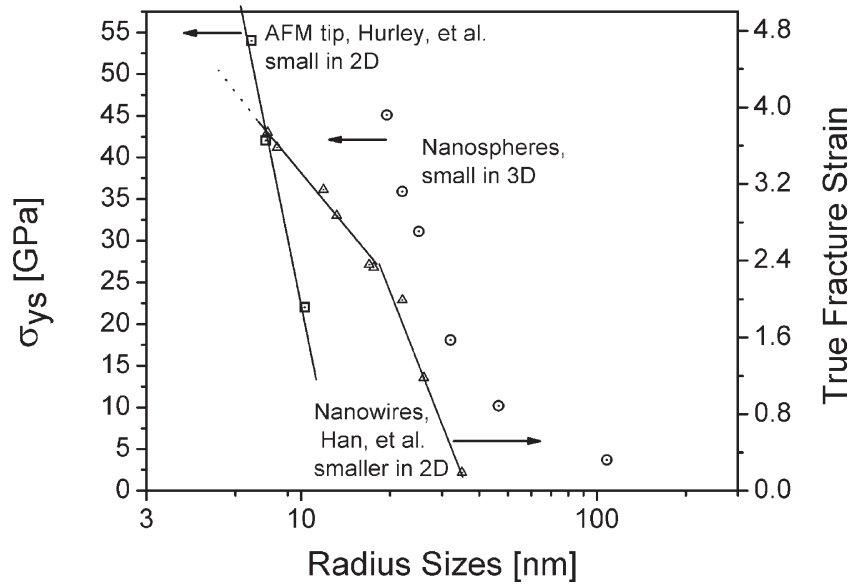

FIG. 1. An illustration of the size effects on mechanical properties as a function of the critical dimension of the material in silicon. First, the yield strength, for both AFM tips, data adapted from Hurley et al. ${ }^{14}$ and for nanospheres, data adapted from Mook et al. ${ }^{27}$ and Gerberich et al. ${ }^{28}$ Additionally, fracture strain shows an increase by a factor of five over a fivefold decrease in the critical particle dimension, in this case wire radius. Wire data adapted from Han et al. ${ }^{17}$ length scale effect to even larger radii. ${ }^{26,27}$ Thus, it appears that there may be both constraints in shape (i.e., no constraint in bulk materials, 1D for thin films, 2D in pillars, and 3D in nanoparticles) as well as size-scale effects in the enhancement of mechanical properties of materials as "brittle" as $\mathrm{Si}$ and $\mathrm{SiC}$. Consider this again for single crystal silicon where stand alone, on chip fracture toughness samples $45 \mu \mathrm{m}$ wide, $100 \mu \mathrm{m}$ long, and $4 \mu \mathrm{m}$ thick were tested with initial crack lengths of $2 \mu \mathrm{m}$. The fracture toughness results shown in Fig. 2 clearly demonstrate a modest brittle to ductile transition only slightly beyond room temperature. Clear dislocation traces in TEM sections after fracture demonstrated deeper plastic zones along the crack path for $300{ }^{\circ} \mathrm{C}$ tests compared to room temperature. ${ }^{28}$ The nearly order of magnitude longer slip traces were qualitatively consistent with the factor of four increase in strain energy release rate. As this was on micron-sized samples, the case can be made from Fig. 1 that this would represent much larger fracture toughness values in submicron structures.

With respect to fatigue, Namazu and Isono $^{25}$ used silicon nanowires tested under cyclic bending by an AFM at $50 \mathrm{~Hz}$ to construct S-N curves. Failure life ranging from $10^{2}$ to $10^{6}$ cycles demonstrated a clear size effect. For single crystal wires $200 \mathrm{~nm}$ wide compared to those $550 \mathrm{~nm}$ wide the number of cycles to failure was a factor of 10 greater at $8 \mathrm{GPa}$ but nearly 100 times greater at $5 \mathrm{GPa}$ peak stresses. This length scale effect is difficult to rationalize by any phase transformation or

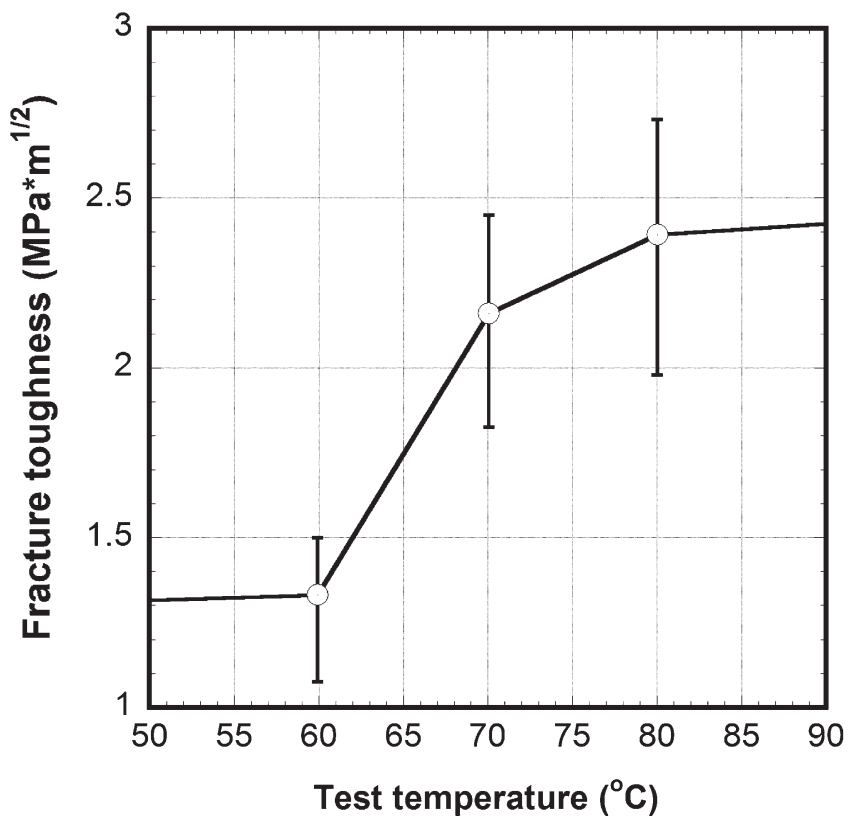

FIG. 2. A brittle to ductile transition at temperatures elevated only slightly above room temperature as evidenced by the reduction of the fracture toughness in micron sized test samples of single crystal silicon. Adapted from S. Nakao et al. ${ }^{29}$ 
oxide-induced crack growth mechanism. However, it is consistent with a dislocation plasticity mechanism as suggested by the length scale effect on true fracture strain shown in Fig. 1.

Altogether, the above size effects on strength, ductility, fracture toughness, and fatigue all point strongly toward dislocation plasticity as the underlying mechanism. This unusual confluence of both strength and ductility increasing with decreasing size scale is reminiscent of the Hall-Petch grain size effect. Here, also, decreased grain size produces increased strength and ductility as well as fracture toughness and fatigue life. If it is as simple as proposed, why has this not been observed previously? The simple answer is that length scales of the magnitude shown in Fig. 1 have not been able to be evaluated in any meaningful way until recently. But then the more complex question is why has improvement in metals and alloys, for example, Hall-Petch, been so successful and not ceramics? Or, why at such small length scales might some ceramics be ductile? The key is that three factors apparently come into play allowing enhanced plasticity at a small scale. First, small volumes, particularly single crystals, are relatively free of stress risers allowing high stresses prior to crack propagation. Second, very high stresses can nucleate dislocations at free surfaces in ceramics at low temperature. Third, the activation energy for nucleating a dislocation is much smaller at high stresses allowing both nucleation and propagation of dislocations to produce plasticity when normalized by structure size. Consider, first, the third case based on dislocation velocity laws grounded upon observations of dislocation velocities at temperatures between approximately $650 \mathrm{~K}$ and $1200 \mathrm{~K}^{29}$ In diamond structure, in particular silicon, the activation energy has been reported as $2.1 \mathrm{eV}$. Using the standard thermal activation model for dislocation velocities, ${ }^{29}$ this is given by

$$
v \approx A \tau^{m} \exp \left(-\frac{Q}{k T}\right),
$$

where $\tau$ is a shear stress, $m$ a power law exponent, and $Q$ an activation energy. One can compare the velocities if $Q \approx 2.1 \mathrm{eV}$ remains constant for temperatures of $573 \mathrm{~K}$ and $300 \mathrm{~K}$. In the above fracture experiments for these two temperatures, TEM images of dislocation depths below the crack plane were imaged after fracture. ${ }^{28}$ Using this activation energy and the local stresses at the crack tip along with stress exponents of 1.6 and 1.95 at $573 \mathrm{~K}$ and $300 \mathrm{~K}$, respectively, the relative velocities were determined. This results in the velocities being 16 orders of magnitude slower at room temperature compared to $573 \mathrm{~K}$. This cannot account for the fact that the observed dislocation depths were only an order of magnitude smaller at room temperature. It seems then that the activation energy must be much smaller. Recently,
$\mathrm{Xu}$ and Zhang ${ }^{30}$ theoretically analyzed dislocation nucleation based upon the Peierls-Nabarro model. They found that the activation energy for dislocation nucleation is much smaller, $0.05 \mu b^{3}$, for the shuffle dislocation Burgers vector which is $a / 2[110]$ giving $Q=1.2 \mathrm{eV}$ using a value of $a_{\mathrm{Si}}=5.43 \AA$ and $\mu_{\mathrm{Si}}=68.1 \mathrm{GPa}^{30}$ Assuming this energy is also pertinent to dislocation velocities this is still too high, as a seven orders of magnitude difference in velocity persists. To explain the observations by the above velocity law, one would have to lower the activation energy to about $0.27 \mathrm{eV}$. This is possible if only one Shockley partial dislocation is considered. As proposed in Ostlund et al. ${ }^{18}$ and $\mathrm{Xu}$ and Zhang, ${ }^{31}$ a size-dependent ductile-to-brittle transition for small features under compression can be explained by the nucleation of a single partial dislocation at the structure's surface. When the structure is small enough, the partial dislocation can glide to the opposing free surface, resulting in shielding of the initial concentrator without nucleating a trailing partial which has a lower mobility. The Burgers vector in this case would be $a / 6$ [112], giving $Q=0.23 \mathrm{eV}$. However, TEM observations after hydrostatic compression in a diamond anvil cell show that full dislocations of the shuffle set have been created. A diamond structure gives rise to two different types of $\{111\}$ glide planes, which in one case leads to widely spaced planes forming the shuffle set with a $\frac{\sqrt{3} a}{4}$ spacing. Alternatively, due to a different shift in the atom positions, glide dislocations are formed with a spacing between planes of $\frac{\sqrt{3} a}{12}$. These tend to dissociate and glide by partial dislocations at high temperatures. While still a matter of controversy, it is now generally believed that low temperature dislocation plasticity consists of undissociated dislocations. While it is clear that dislocation plasticity is the mechanism, the exact details for each specific load case, for example, magnitude and state of stress, remain an open question.

Once the activation energy for the thermally activated process is resolved, the other two features of high stresses and the dislocation nucleation process follow. Regarding the latter, an extensive overview addresses the dislocation nucleation process in detail. ${ }^{11}$ Here, it is discussed that small activation volumes ranging from about $2 b^{3}$ to $20 b^{3}$ are involved in the dislocation nucleation process for silicon nanospheres ranging from 20 to $200 \mathrm{~nm}$ in radius. This is shown to be consistent with classical nucleation ${ }^{29}$ theory as well as thermally activated processes ${ }^{32,33}$ which seems likely given the above discussion on temperature effects.

It is proposed then that high stresses may nucleate dislocations at the surface of small volumes of brittle materials well below the stress that is necessary for small but sparse inherent defects to nucleate fracture. This leads to the dislocation-shielding concept depicted in Fig. 3. For example, a surface step on an oxide single 


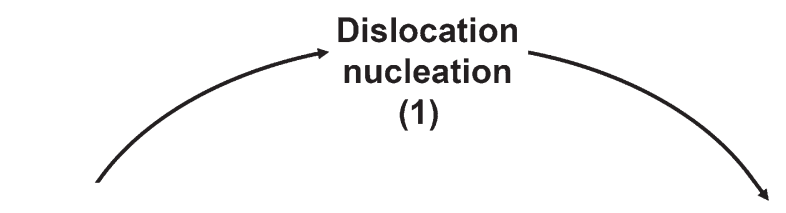

Increased
strength

(2) Shielding

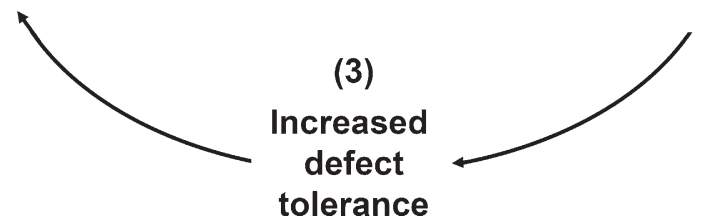

FIG. 3. A cyclic process in which the initial dislocation nucleation creates dislocation shielding, which in turn increases the defect tolerance, and results in increasing strength. The process then repeats until fracture.

crystal may represent a stress concentration that could nucleate either dislocations or fracture. A step even $2 \mathrm{~nm}$ high, if considered as a crack length, would require a stress of $25 \mathrm{GPa}$ to trigger fracture in a typical ceramic having $K_{\mathrm{Ic}} \approx 2 \mathrm{MPa} \cdot \mathrm{m}^{1 / 2}$. However, at stresses much lower than that, such a stress riser would easily allow dislocation nucleation. Page et al. ${ }^{34}$ clearly demonstrated that to be the case in $\mathrm{SiC}$ a decade ago. As indicated in Fig. 3, dislocation nucleation would lead to shielding of that particular stress riser giving increased defect tolerance. This in turn allows higher stresses resulting in an increased strength. But these higher stresses may produce additional dislocations and navigating this circular path multiple times would produce both higher strength and higher ductility prior to incipient fracture.

\section{EXPERIMENTAL SCALE EFFECTS IN SILICON AND SAPPHIRE}

As suggested in the schematic of Fig. 3, dislocation shielding of potential fracture critical stress concentrators or cracks results in increased strength. How does this scale with size? With shielding dislocations allowing higher strength prior to fracture, a crude relation was previously derived to show that fracture toughness was given by ${ }^{36}$

$$
K_{\mathrm{Ic}}=\frac{4}{3} \sqrt{\frac{N \mu \sigma_{\mathrm{ys}} b}{1-v}},
$$

for $N$ shielding dislocations with $\mu \sigma_{y s} b$ being the product of shear modulus, yield strength and Burgers vector and $v$, Poisson's ratio. Eq. (2) comes from a crack extension force correlation, as explained elsewhere in detail, ${ }^{36}$ wherein the dislocation force per unit length acts as a shielding force per unit length. From a crack growth resistance standpoint, this is balanced by the strain energy release rate. The latter has been traditionally considered as a crack extension force per unit length of the crack with $\mathrm{J} / \mathrm{m}^{2} \equiv \mathrm{N} / \mathrm{m}$. This predicted the fracture toughness of 22 relatively brittle semiconductors, oxides, nitrides, carbides, and silicides with a small scatter band around $N=14 .{ }^{36}$ Most fracture toughness results were from indentation into single crystals or largegrain polycrystals. Using this to relate yield strength to a length scale, a constitutive relationship between stress and strain as related to displacement is needed. For these "brittle" materials with few dislocations involved, a first-order, elastic relationship gives

$$
\sigma=E \varepsilon=E \frac{\delta}{\alpha r}
$$

where displacement is $\delta$, the structure radius is $r$, and $\alpha$ is a geometrical parameter that may be structure dependent. For nanosphere compression, the best fit was found using a value of $\alpha=4$. Since the initial height for the nanosphere compression is the sphere diameter $(2 r)$, and for the pillar compression is the pillar height which has a $3: 1$ aspect ratio $(6 r)$, the value of $\alpha$ for pillar geometry $\left(\alpha_{\text {pillar }}\right)$ is expected to be three times the value of $\alpha$ for sphere geometry $\left(\alpha_{\text {sphere }}\right)$. To demonstrate the relationship between fracture toughness and yield stress, the plot is reshown here in Fig. 4(a), which gives the fit to Eq. (2). Note that for the largest scatter of AlN, the two data points from the literature cited ${ }^{36}$ were about equidistant from the prediction. What was not shown in that paper and found after this graph was published is the TEM observation in Fig. 4(b). The arrested crack in single crystal silicon does have 14 shielding dislocations at its tip in Fig. 4(b). ${ }^{35}$ As the orientation, test conditions, and so on, are not known, this exact match is fortuitous noting that the silicon data point does not fall on the line in Fig. 4(a). Still, it is clear that dislocation shielding for such materials is a valid concept. Furthermore, the displacement at yielding, $\delta$, can be taken as $N b$ dislocations which with $E=2(1+v) \mu$ and Eqs. (2) and (3) leads to a relationship between yield stress and fracture toughness. This is given by

$$
\sigma_{\mathrm{ys}} \approx \frac{3}{4} K_{\mathrm{Ic}}\left(\frac{2\left(1-v^{2}\right)}{\alpha r}\right)^{1 / 2} .
$$

While we have taken some license with describing the dislocation number and the constitutive relationship, Eq. (4) qualitatively describes the relationship between yield strength and fracture toughness. ${ }^{36}$ This recursive relationship can once again use Eq. (2) to eliminate $K_{\text {Ic }}$ with $N=\delta / b$ as before giving

$$
\sigma_{\mathrm{ys}}=\frac{2 \mu(1+v) \delta}{\alpha r} .
$$

If roughly the same amount of dislocation shielding occurs prior to fracture for a given material under a specific loading condition, then picking $\delta$ as a constant 


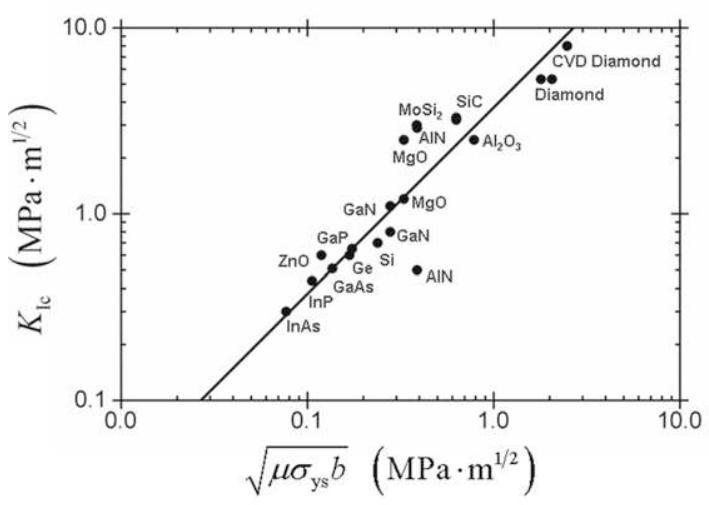

(a)

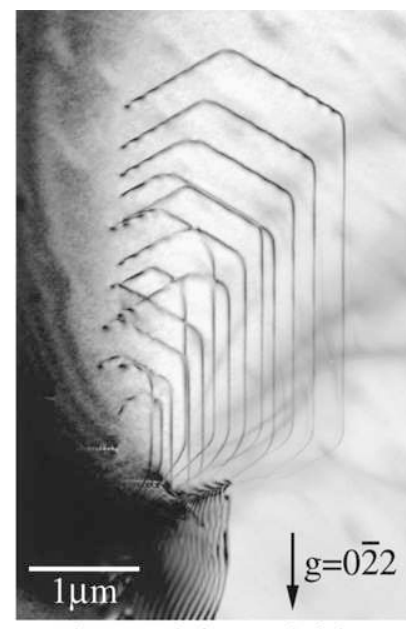

Tanaka \& Higashida Journal of Electron Microscopy 200454 (4) 353-360

(b)

FIG. 4. (a) A fit of 22 ceramic materials by Eq. (2), relating the fracture toughness to the number of shielding dislocations, in this case $N=14$, the shear modulus, Poisson's ratio, the Burger's vector, and the yield strength. Data adapted from Gerberich et al. ${ }^{35}$ (b) Tanaka and Higashida ${ }^{36}$ show an arrested crack with $N=14$ shielding dislocations in single crystal silicon with unknown test conditions.

gives a size dependent yield stress or flow stress. Fewer dislocations would give the same shielding the more confined the structure is, that is, one-dimensional versus two dimensional for the same radius. Therefore, $\delta=$ $5 \mathrm{~nm}$ was used for the nanospheres and $30 \mathrm{~nm}$ for the nanopillars in Fig. 5. With these values for $\delta / \alpha$, the firstorder length-scale relationship of Eq. (5) does account for the scale dependency of these structures for silicon. Checking back to the actual displacements at which failure occurred gave average values of $23 \pm 10 \mathrm{~nm}$ and 300 $\pm 50 \mathrm{~nm}$ for the spheres and the pillars with a diameter smaller than $2 \mu \mathrm{m}$, respectively. The factor $\alpha_{\text {sphere }}=4$ for the spheres and thus $\alpha_{\text {pillar }}=12\left(\alpha_{\text {pillar }}=3 \alpha_{\text {sphere }}\right)$ for the pillars used with Eq. (5) is consistent with the above approximations. It also represents a consistent approach and basis for the strength dependence at very small length scales. It is recognized that this first-order relationship represents data trends, but is not a particularly good fit to much of the data. First, Eq. (5) has only one fitting parameter, $\alpha$, for all cases, which may not reflect the geometric differences between spheres and nanopillars, beams, or nanoindentation. The data from Namazu et al. ${ }^{24}$ represents the bending of silicon beams, that from Moser et al., ${ }^{15}$ the compression of silicon nanopillars, from Mook et al., ${ }^{26}$ the compression of silicon nanospheres, and from Stauffer et al. ${ }^{37}$ for nanoindented sapphire such that the equivalent radius is the contact radius. As such, there are major differences in material, state of stress, and scale factors. For example, the amount of elastic displacement in a tall thin column or a bent beam would be much greater prior to a plastic strain than a constrained nanosphere. Using a variational boundary integral formulation of the Peierls-Nabarro

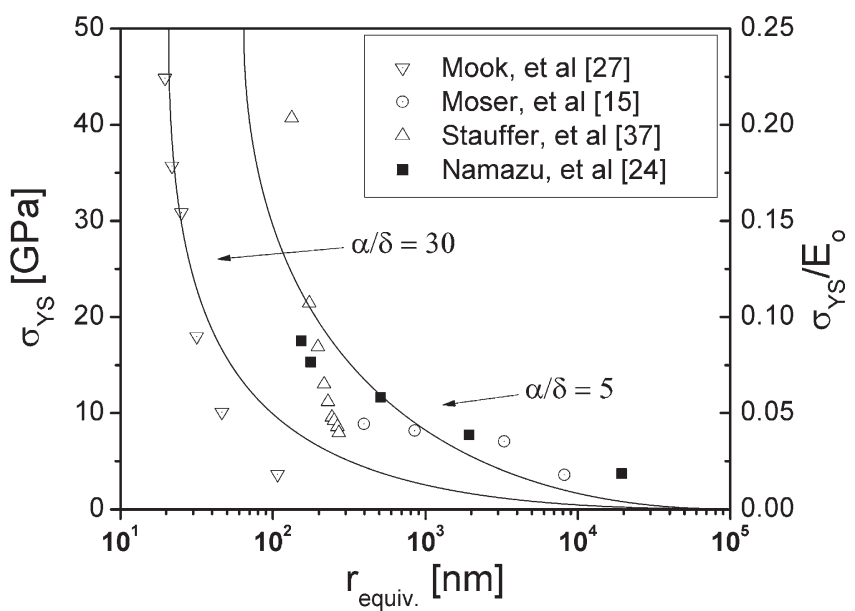

FIG. 5. On the left, the yield strength for silicon objects with a critical nanometer sized dimension taken as a function of the equivalent radius. Predictions from Eq. (5) are for constant $\delta / \alpha$ equal to $5 \mathrm{~nm}$ and $30 \mathrm{~nm}$. On the right, the yield strength over the bulk modulus as a function of the equivalent radius in single crystal c-axis sapphire. Data for silicon adapted from Namazu et al., ${ }^{24}$ Moser et al., ${ }^{15}$ and Mook et al. ${ }^{27}$ Data for sapphire is unpublished from Stauffer et al. ${ }^{37}$

dislocation model, Zhang and $\mathrm{Xu}^{30,38}$ derived a different dependence. Based on the critical condition of dislocation nucleation under a flat punch, they propose the hardening effect to be proportional to the inverse square root of the indenter width. This would represent a milder dependence of strength on size, which could represent what is found in the micron scale but not at the smallest scales. Data in Fig. 5 on single crystal sapphire are included to show that this phenomenon is not limited to a single material.

With the above strength dependence on length scale, it is next appropriate to demonstrate how 
fracture toughness, $K_{\text {Ic }}$, or strain energy release rate, $G_{I c}=K_{\mathrm{Ic}}^{2} / E\left(1-v^{2}\right)$, varies with the same length scale. For nanospheres, previously published ${ }^{1} K_{\text {Ic }}$ values were experimentally evaluated in the $20 \mathrm{~nm}$ to $100 \mathrm{~nm}$ radius range. Considerably larger nanopillars ${ }^{15}$ allowed a calculation of $G_{\text {Ic }}$ based upon a strain energy density model and the activation volume, $V^{*}$, for dislocation nucleation. The model will be addressed in the next section. The strain energy density was taken as $\sigma_{\text {flow }} \varepsilon$, with the observed flow stress and strain at maximum load. As will be shown in a forthcoming paper ${ }^{18}$ there is a transition between 310 to $400 \mathrm{~nm}$ where nanopillars with diameters greater than that tend to fracture, which can be seen in Fig. 6. Pillars below this transition represent a lower bound. Additionally, it was possible to determine $G_{\text {Ic }}$ for nanowires tested in bending ${ }^{24}$ using the same analytical procedure. Two major differences were involved in that the wires had a trapezoidal shape for which an equivalent circular radius was calculated having the same cross-sectional area for a given test section. The second difference was that the strength reported was the maximum bending stress at the top section which would be greater than average whereas the nanopillars were under uniform compression. Third, the processing conditions were different as reported elsewhere. ${ }^{15,24}$ In both cases the activation volume, $V^{*}$, is required and this was estimated from an inverse relationship between yield or flow stress and $V^{*} .{ }^{11,38}$ Observed values of $\mathrm{G}_{I c}$ for the nanospheres and calculated values based upon strain energy density for pillars and wires are shown as a function of inverse radius in Fig. 7. Over a size range of two orders of magnitude plotted as inverse radius, the strain energy release rate increased by about a factor of two. Below a $100 \mathrm{~nm}$ radius, however, the $G_{\text {Ic }}$ values for the nanospheres increased by about a factor of eight in less than a decade of size scale. This behavior is consistent with the steep rise in strength and ductility shown in Fig. 1 for length scales below $100 \mathrm{~nm}$.

\section{FRACTURE CONCEPT BASED ON DISLOCATION NUCLEATION}

The shielding concept for enhanced fracture is that parallel slip bands of $n_{s}$ dislocations develop in

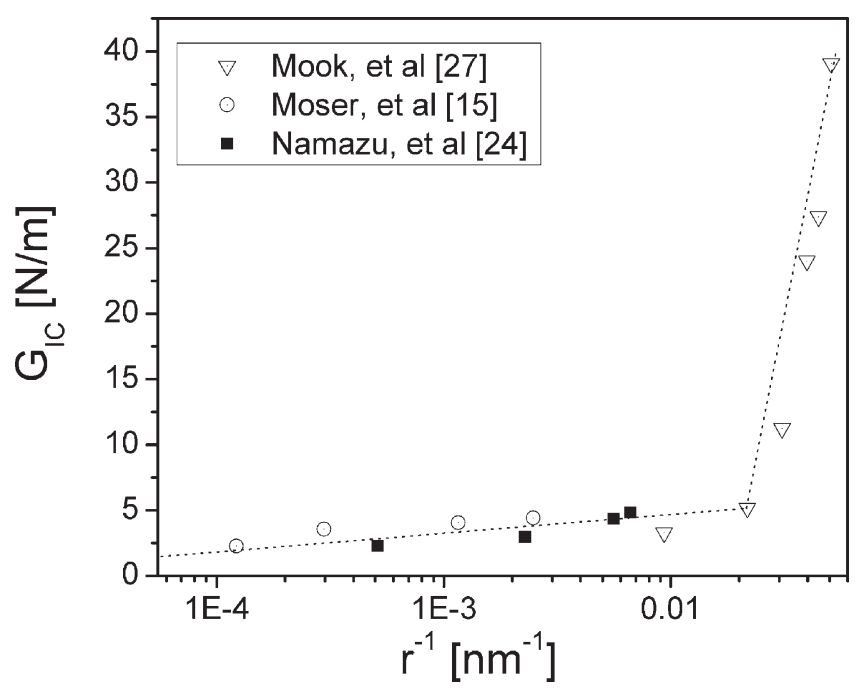

FIG. 7. Strain energy density as a function of the inverse radius for silicon wires, pillars, and nanospheres. Note the change in the behavior for systems greater than $100 \mathrm{~nm}$ radius.

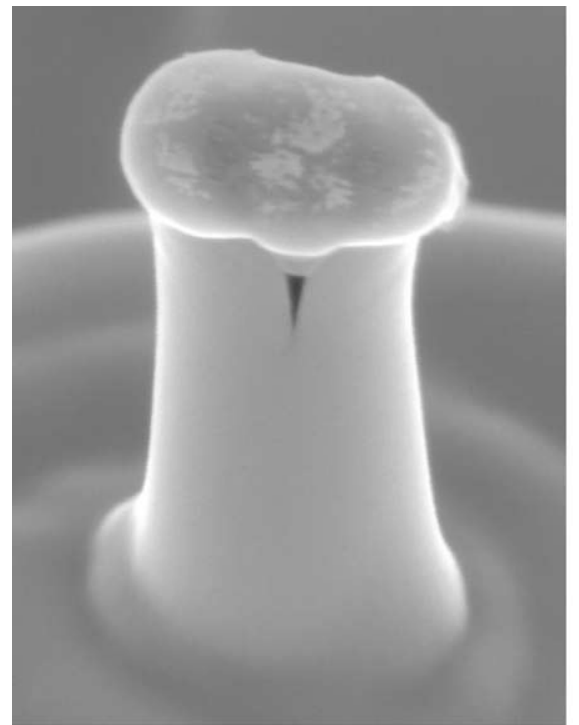

(a)

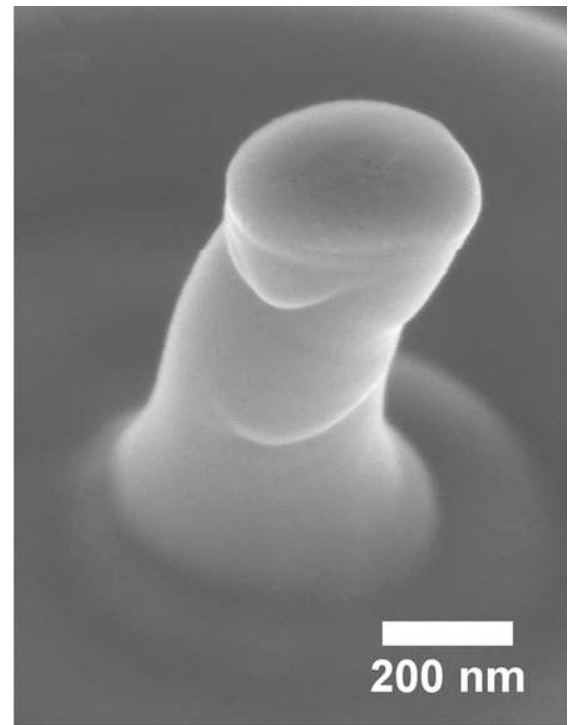

(b)

FIG. 6. Silicon pillars fabricated by FIB milling and compressed with an MTS Nanoindenter XP imaged at $40^{\circ}$ by SEM. (a) This pillar has a diameter of $400 \mathrm{~nm}$ and developed a vertical crack while the pillar in (b) has a diameter of $310 \mathrm{~nm}$ and deformed plastically. ${ }^{18}$ 
relatively small, brittle volumes similar to, but fewer than, those observed by Lloyd et al., ${ }^{40}$ in large indentations of spinel. As previously described, the spacing, $\ell_{\mathrm{w}}$, of these is related to an activation volume, giving ${ }^{39}$

$$
V^{*} \approx b^{2} \ell_{\mathrm{w}}
$$

For a total number of shielding dislocations, $N$, on a number of parallel slip planes given by $2 a / \ell_{\mathrm{w}}$ where $a$ is the contact radius, the number on one plane would be

$$
n_{s}=\frac{N \ell_{\mathrm{w}}}{4 a} \text {. }
$$

since the total contact length would be $2 a$ and the plastic zone diameter would extend to $4 a$. A detailed analysis of the relationship between the plastic zone and the contact radius can be found in the Appendix. By eliminating $\ell_{\mathrm{w}}$ from Eqs. (6) and (7) and substituting normalized displacement, $\delta / b$, for $N$ dislocations, one finds

$$
\mathrm{n}_{\mathrm{s}}=\frac{V^{*}}{b^{2}} \cdot \frac{\delta}{b} \cdot \frac{1}{4 a}
$$

The strain associated with this number is taken as the Tabor strain, $\varepsilon=0.2 a / r$ and since $a \approx \sqrt{\delta r}$ at the top and bottom of a sphere, $\varepsilon=0.2(\delta / r)^{1 / 2}$. But $\delta / a=\sqrt{\delta / r}$, allowing an elimination of $\delta / a$ in Eq. (8) by $5 \varepsilon$, giving for one plane

$$
n_{\mathrm{s}}=\frac{1.25 V^{*} \varepsilon}{b^{3}}
$$

as derived somewhat differently elsewhere. ${ }^{11}$ As was determined previously ${ }^{36}$ a fracture toughness from a simple dislocation shielding argument is given by Eq. (2). This can be put in terms of Young's modulus using $\mu=E / 2(1+v)$ and a strain energy release rate, $G_{\mathrm{Ic}}=K_{\mathrm{Ic}}^{2} / E\left(1-v^{2}\right)$. For Poisson's ratio, $v=0.3$, these give with Eqs. (2) and (9) a lower bound estimate for $G_{\text {Ic }}^{L}$ at $n_{\mathrm{s}}=N$, which is

$$
G_{\mathrm{Ic}}^{\mathrm{L}}=\frac{4}{3} \frac{\sigma_{\mathrm{ys}} \varepsilon V^{*}}{b^{2}} .
$$

While some license was taken with how numbers and the distribution of dislocations might be related to displacement, subsequent $2 \mathrm{D}$ and 3D analyses should only change the coefficient in Eq. (10). This appears to be a fundamental relationship for small volumes where fracture resistance is coupled to the strength and dislocation shielding as proposed in Fig. 3. A way of viewing this is that the strain increases until a critical strain energy density times the activation volume for dislocation nucleation is sufficient for bond breakage creating an instability. A simpler alternative view is that this is the local work per unit fracture area. To connect this scale to continuum, if possible, one of the reviewers wondered if a length scale parameter as suggested by Atkins and $\mathrm{Mai}^{41}$ might apply. They proposed that a characteristic length parameter was represented by the toughness to strength ratio. Since these values were available for the nanospheres and were available or could be calculated for the nanopillars a ratio using the experimental values was determined for the nanospheres and compared to the theoretical values of Eq. (10). The values observed and calculated were $0.76 \pm 0.2 \mathrm{~nm}$ and $0.73 \pm 0.55 \mathrm{~nm}$, respectively. For the nanopillars in Fig. $7, G_{\mathrm{IC}}$ had been calculated from Eq. (10) so that the determination of a length scale is less significant. Still, the values of $G_{\mathrm{IC}}$ in Fig. 7 for nanopillars converts to $K_{\mathrm{IC}}$ values of $0.76 \pm 0.13 \mathrm{MPa} \cdot \mathrm{m}^{1 / 2}$ which seem realistic for $\mathrm{Si}$. Using the calculated values, $G_{\mathrm{IC}} / \sigma_{\mathrm{ys}}$ gave $0.56 \pm 0.15$ $\mathrm{nm}$. Whether this difference between spheres and pillars is significant or not remains to be seen. It is interesting that this length scale, at the nanoscopic scale, is only slightly greater than the silicon lattice spacing of $0.543 \mathrm{~nm}$. To illustrate the pathway to improved toughness in semiconductors, it is instructive to use Eq. (10) with data that can estimate plastic energy dissipation in the small structures of Fig. 1. This had been qualitatively illustrated for the silicon nanospheres since both work per unit fracture area and $K_{\text {Ic }}$ values have been previously determined. ${ }^{26}$ To demonstrate the size scale effect, it is useful to convert activation volume to a length scale times $b^{2}$. Here the radius, $r$, is used rather than half the circumference of a sphere or nanowire as the contact length for dislocation nucleation would be relatively small and this gave a good comparison to independently measured activation volumes. ${ }^{39}$ From Eq. (10), this gives

$$
G_{\mathrm{Ic}}^{\mathrm{L}}=\frac{4}{3} \sigma_{\mathrm{ys}} \varepsilon r
$$

Even though this appears to have strain energy release rate increasing with size, at the smallest scales the opposite is true since both yield strength and fracture ductility increase with inverse radius. Recalling Fig. 1, it was shown in the range of 5 to $30 \mathrm{~nm}$ that large increases in both yield stress and true fracture strain were determined for the silicon AFM tips and nanowires. The overlap region was used to determine $\sigma_{\mathrm{ys}} \varepsilon$ and knowing $r$ values, $G_{\mathrm{Ic}}^{\mathrm{L}}$ could be calculated. By converting this to $K_{\mathrm{Ic}}$, this could be compared to the previously measured $K_{\text {Ic }}$ values of nanospheres as a function of inverse radius. The remarkable result in Fig. 8 suggests that large fracture toughness values may be achieved at small scales in semiconductors and perhaps in ceramics as well. It may be noted that the slope for the nanospheres, which are constrained in three dimensions, may be even larger than the nanowires, which are constrained in two dimensions. However, the data are sufficiently sparse at this juncture to say that the separation in Fig. 1 ostensibly due to shape will persist. This would be a worthwhile investigation to pursue. 


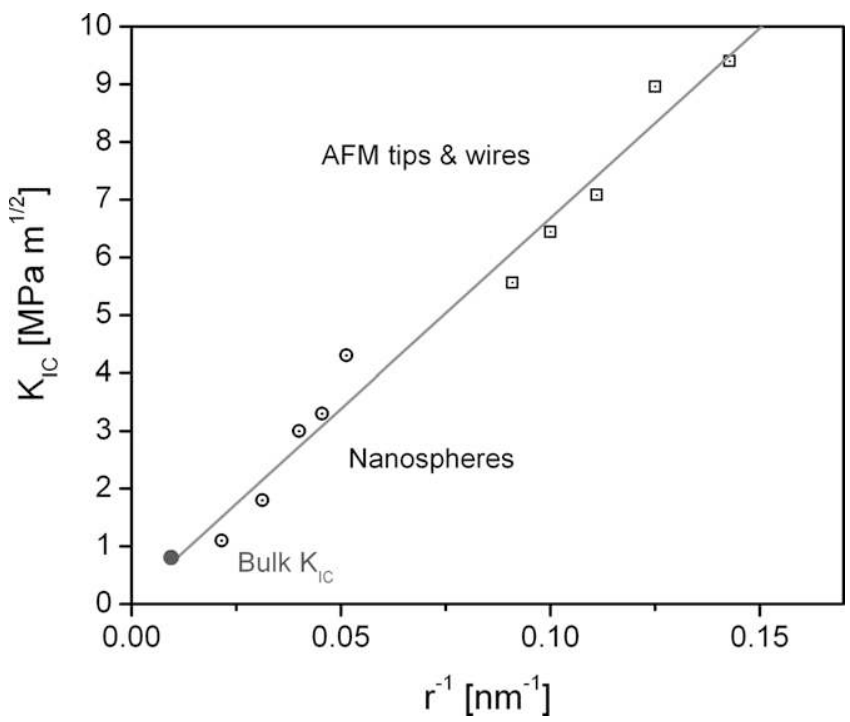

FIG. 8. Fracture toughness converted from the strain energy density for nanosized silicon objects showing a 10 -fold increase in the fracture toughness from that of bulk silicon as the critical radius decreases.

\section{SUMMARY}

What has been shown experimentally is that large scale effects in strength, ductility, and fracture toughness exist in semibrittle silicon. For length scales under several hundred nanometers, properties may increase by an order of magnitude. A dislocation-shielding model is proposed to be responsible and can model to first order both increasing strength and fracture toughness at decreasing scales. The key to the process is dislocation nucleation at surface sites, which shields incipient fracture sites from instability.

\section{ACKNOWLEDGMENTS}

This research was funded through NSF Grant No. CMS-0322436 and the NSF IGERT program through DGE-0114372. Parts of this work were carried out in the University of Minnesota I.T. Characterization Facility, which receives partial support from NSF through the NNIN program. We also appreciate support for one of us (D.D.S.) from Hysitron, Inc.

\section{REFERENCES}

1. W.D. Nix and H. Gao: Indentation size effects in crystalline materials: A law for strain gradient plasticity. J. Mech. Phys. Solids 46, 411 (1998).

2. E. Arzt: Overview No. 130 - Size effects in materials due to microstructural and dimensional constraints: A comparative review. Acta Mater. 46, 5611 (1998).

3. S.G. Corcoran, R.J. Colton, E.T. Lilleodden, and W.W. Gerberich: Anomalous plastic deformation at surfaces: Nanoindentation of gold single crystals. Phys. Rev. B: Condens. Matter 55, 16057 (1997).
4. K.J. Hemker and W.W. Sharpe, Jr.: Microscale characterization of mechanical properties. Annu. Rev. Mater. Sci. 37, 93 (2007).

5. A. Gouldstone, N. Chollacoop, M. Dao, J. Li, A. Minor, and Y-L. Shen: Indentation across size scales and disciplines: Recent developments in experimentation and modeling. Acta Mater. 55, 4015 (2007)

6. K. Durst, B. Backer, O. Franke, and M. Goker: Indentation size effect in metallic materials: Modeling strength from pop-in to macroscopic hardness using geometrically necessary dislocations. Acta Mater. 54, 2547 (2006).

7. T. Tsuchiya, O. Tabata, J. Sakata, and Y. Taga: Specimen-size effect on tensile strength of surface-micromachined polycrystalline silicon thin film. J. Microelectromech. Syst. 1, 106 (1998).

8. C.C. Koch, I.A. Ovid'ko, S. Seal, and S. Veprek: Structural Nanocrystalline Materials (Cambridge University Press, Cambridge, UK, 2007), Ch. 4.

9. H. vanSwygenhoven and J.R. Weertman: Deformation in nanocrystalline metals. Mater. Today 9, 24 (2006).

10. W.W. Gerberich, N.I. Tymiak, J.C. Grunlan, M.F. Horstemeyer, and M.I. Baskes: Interpretations of indentation size effects J. Appl. Mech. 69, 433 (2002).

11. W.W. Gerberich, D.D. Stauffer, A.R. Beaber, and W.M. Mook: Connectivity between plasticity and brittle fracture: An overview from nanoindentation studies. Proc. J. Mech. E 222, 1 (2009).

12. J.R. Greer and W.D. Nix: Size dependence of mechanical properties of gold at the submicron scale. Appl. Phys. A 80, 1625 (2005).

13. M.D. Uchic, D.N. Dimiduk, J.N. Florando, and W.D. Nix: Sample dimensions influence strength and crystal plasticity. Science 305, 986 (2004).

14. M. Kopycinska-Mueller, R.H. Geiss, and D.C. Hurley: Sizerelated plasticity effects in AFM silicon contilever tips, in Mechanics of Nanoscale Materials and Devices, edited by A. Misra, J.P. Sullivan, H. Huang, K. Lu, and S. Asif (Mater. Res. Soc. Symp. Proc. 924E, Warrendale, PA, 2006), 0924-Z03-02.

15. B. Moser, K. Wasmer, L. Barbieri, and J. Michler: Strength and fracture of $\mathrm{Si}$ micropillars: A new scanning electron microscopy-based micro-compression test. J. Mater. Res. 22, 1004 (2007).

16. W.M. Mook, M.S. Lund, C. Leighton, and W.W. Gerberich: Flow stresses and activation volumes for highly deformed nanoposts. Mater. Sci. Eng., A 493, 12 (2008).

17. X. Han, K. Zheng, Y.F. Zhang, X. Zhang, Z. Zhang, and Z.L. Wang: Low temperature in situ large-strain plasticity of silicon nanowires. Adv. Mater. 19, 2112 (2007).

18. F. Ostlund, K. Rzepiejewska-Malyska, K. Leifer, and J. Michler: Brittle-to-ductile transition in uniaxial compression of silicon pillars at room temperature. Adv. Mater. (2009 submitted).

19. T.Y. Kim, S.S. Han, and H.M. Lee: Nanomechanical behavior of $\beta$-SiC nanowire in tension: Molecular dynamic simulations. Mater. Trans. 45, 1442 (2004).

20. X.D. Han, Y.F. Zhang, K. Zheng, X.N. Zhang, Y. Hao, X.Y. Guo, J. Yuan, and Z.L. Wang: Low-temperature in situ large strain plasticity of ceramic $\mathrm{SiC}$ nanowires and its atomic-scale mechanism. Nano Lett. 7, 452 (2007).

21. K. Kang and W. Cai: Brittle and ductile fracture of semiconductor nanowires. Molecular dynamics simulations. Philos. Mag. 87, 2169 (2007).

22. P. Gumbsch, S. Taeri-Baghbadruni, D. Brunner, W. Sigle, and M. Ruhle: Plasticity and an inverse brittle-to-ductile transition in strontium titanate. Phys. Rev. Lett. 87, 085505 (2001).

23. Y. Zhang, X. Han, K. Zheng, Z. Zhang, X. Zhang, J. Fu, Y. Ji, Y. Hao, X. Guo, and Z.L. Wang: Direct observation of superplasticity of $\beta$-SiC nanowires at low temperature. Adv. Funct. Mater. 17, 3435 (2007). 
24. T. Namazu, Y. Isono, and T. Tanaka: Nano-scale bending test of $\mathrm{Si}$ beam for MEMS, in Annual International Conference on MEMS 2000 (IEEE, Piscataway, NJ, 2000), pp. 205-210.

25. T. Namazu and Y. Isono: High-cycle fatigue test of nanoscale $\mathrm{Si}$ and $\mathrm{SiO}_{2}$ wires based on AFM technique, in Annual International Conference on MEMS 2003 (IEEE, Piscataway, NJ, 2003), pp. 662-665.

26. W.M. Mook, J.D. Nowak, C.R. Perrey, C.B. Carter, R. Mukherjee, S.L. Girshick, P.H. McMurry and W.W. Gerberich: Compressive stress effect on nanoparticle modulus and fracture. Phys. Rev. B 75, 1 (2007)

27. W.W. Gerberich, W.M. Mook, C.R. Perrey, C.B. Carter, M.I. Baskes, R. Mukherjee, A. Gidwani, J. Heberlein, P.H. McMurry, and G.L. Girshick: Superhard silicon nanospheres. J. Mech. Phys. Solids 51, 979 (2003).

28. S. Nakao, T. Ando, M. Shikida, and K. Sato: Effects of temperature on fracture toughness in a single-crystal-silicon film and transition in its fracture mode. J. Micromech. and Microeng. 18, 1 (2008).

29. P. Haasen, U. Messerschmidt, and W. Skrotzki: Low-energy dislocation structures in ionic crystals and semiconductors. Mater. Sci. Eng. 81, 493 (1986).

30. G. Xu and C. Zhang: Analysis of dislocation nucleation from a crystal surface based on the Peierls-Nabarro dislocation model. J. Mech. Phys. Solids 51, 1371 (2003).

31. J. Michler, K. Wasmer, S. Meier, F. Ostlund, and K. Leifer: Plastic deformation of gallium arsenide micropillars under uniaxial compression at room temperature. Appl. Phys. Lett. 90(4), 1 (2007).

32. C.A. Schuh, J.K. Mason, and A.C. Lund: Quantitative insight into dislocation nucleation from high temperature nanoindentation experiments. Nat. Mater. 4, 617 (2005).

33. T. Zhu, J. Li, A. Samata, A. Leach, and K. Gall: Temperature and strain-rate dependence of surface dislocation nucleation. Phys. Rev. Lett. 100, 1 (2008).

34. T.F. Page, L. Riester, and S.V. Hainsworth: The plasticity response of $6 \mathrm{H} \mathrm{SiC}$ and related isostructural materials to nanoindentation: Slip vs. densification, in Fundamentals of Nanoindentation and Nanotribology, edited by N.R. Moody, W.W. Gerberich, N. Burnham, and S.P. Baker (Mater. Res. Soc. Symp. Proc. 522, Warrendale, PA, 1998), pp. 113-118.

35. M. Tanaka and K. Higashida: HVEM characterization of crack tip dislocations in silicon crystals. J. Electron Microsc. (Tokyo) 53 (4), 353 (2004).

36. W.W. Gerberich, W.M. Mook, C.B. Carter, and R. Ballarini: A crack extension force correlation for hard materials. Int. J. Fract. 148, 109 (2007).

37. D.D. Stauffer, A. Beaber, and W.W. Gerberich: Unpublished Data, University of Minnesota.
38. C. Zhang and G. Xu: Energetics of dislocation nucleation under a nanoindenter. Mater. Sci. Eng., A 400-401, 471 (2005).

39. M.J. Cordill, N.R. Moody, and W.W. Gerberich: The role of dislocation walls for nanoindentation to shallow depths. Int. J. Plast. 25, 281 (2009).

40. S.J. Lloyd, A. Casterello, F. Guiliani, Y. Long, K.K. McLaughlin, J.M. Molina-Aldaregula, N.A. Stelmashenko, J.L. Vandepere, and W.J. Clegg: Observations of nanoindents via cross-sectional transmission electron microscopy: A survey of deformation mechanisms. Proc. R. Soc. London, Ser. A 461, 2521 (2005).

41. A.G. Atkins and Y.W. Mai: Elastic and Plastic Fracture: Metals, Polymers, Ceramics, Composites, Biological Materials (Ellis Horwood, Chichester; Halsted Press, New York, 1985).

42. K.L. Johnson: Contact Mechanics (Cambridge University Press, Cambridge, UK, 1985, reprinted 2001), pp. 174-178.

43. S. Harvey, H. Huang, S. Venkataraman, and W.W. Gerberich: Microscopy and microindentation mechanics of single crystal Fe $-3 \mathrm{wt} \%$ Si: Part I. Atomic force microscopy of a small indentation. J. Mater. Res. 8, 1291 (1993).

44. F.J. Lockett: Indentation of a rigid/plastic material by a conical indenter. J. Mech. Phys. Solids 11, 345 (1962).

\section{APPENDIX}

This appendix gives an analysis of the ratio of the plastic zone radius, $c$, to the contact radius, $a$.

The ratio of the plastic zone radius, $c$, to the contact radius, $a$, depends on the shape of the indenter and the plasticity model. For Johnson's spherical cavity model in the fully plastic state with mean pressure (hardness, $H$ ) to yield strength equal to 3 , a value of 2.3 is obtained for $c / a{ }^{42}$ On the other hand, we have shown that the plastic zone, using Johnson's approach, can be given by ${ }^{43}$

$$
c=\sqrt{\frac{3 P}{2 \pi \sigma_{\mathrm{ys}}}} .
$$

Taking the same condition that $H \approx 3 \sigma_{\mathrm{ys}}=P / \pi a^{2}$, it is easily shown that $c=2.12 a$ from Eq. (A1). Finally, with a slip line solution from Lockett ${ }^{44}$ it was previously reported $^{42}$ for a conical indenter with a $70^{\circ}$ or $80^{\circ}$ contact angle and the von Mises yield criterion that $c / a \approx 1.53$. Coincidentally, the average of these three values give $c / a \approx 2$ as used in Eq. (7). 